St., New York, New York 10032, USA. Phone: 212.305.3720; Fax: 212.305.3545; E-mail: rsk20@columbia.edu.

1. Fischer TZ, Waxman SG. Familial pain syndromes from mutations of the NaV1.7 sodium channel. Ann N Y Acad Sci. 2010;1184:196-207.

2. Ruan Y, Liu N, Priori SG. Sodium channel mutations and arrhythmias. Nat Rev Cardiol. 2009; 6(5):337-348.

3. Catterall WA, Dib-Hajj S, Meisler MH, Pietrobon D. Inherited neuronal ion channelopathies: new windows on complex neurological diseases. J Neurosci. 2008;28(46):11768-11777.

4. Belardinelli L, Shryock JC, Fraser H. Inhibition of the late sodium current as a potential cardioprotective principle: effects of the late sodium current inhibitor ranolazine. Heart. 2006;92(suppl 4):iv6-iv14.

5. Fozzard HA, Lee PJ, Lipkind GM. Mechanism of local anesthetic drug action on voltage-gated sodium channels. Curr Pharm Des. 2005;11(21):2671-2686.

6. Bankston JR, Kass RS. Molecular determinants of local anesthetic action of beta-blocking drugs: Implications for therapeutic management of long QT syndrome variant 3. J Mol Cell Cardiol. 2010;48(1):246-253.

7. Fredj S, Sampson KJ, Liu H, Kass RS. Molecular basis of ranolazine block of LQT-3 mutant sodium channels: evidence for site of action. Br J Pharmacol. 2006;148(1):16-24

8. Undrovinas A, Maltsev VA. Late sodium current is a new therapeutic target to improve contractility and rhythm in failing heart. Cardiovasc Hematol Agents Med Chem. 2008;6(4):348-359.

9. Sampson KJ, Kass RS. Molecular mechanisms of adrenergic stimulation in the heart. Heart Rhythm. 2010;7(8):1151-1153.

10. Carnegie GK, Means CK, Scott JD. A-kinase anchoring proteins: from protein complexes to physiology and disease. IUBMB Life. 2009;61(4):394-406.

11. Abriel H, Kass RS. Regulation of the voltage-gated cardiac sodium channel Nav1.5 by interacting proteins. Trends Cardiovasc Med. 2005;15(1):35-40.

12. Abriel H. Cardiac sodium channel $\mathrm{Na}(\mathrm{v}) 1.5$ and interacting proteins: Physiology and pathophysi- ology. J Mol Cell Cardiol. 2010;48(1):2-11.

13. Hedley PL, et al. The genetic basis of long QT and short QT syndromes: a mutation update. Hum Mutat. 2009;30(11):1486-1511.

14. Hedley PL, et al. The genetic basis of Brugada syndrome: a mutation update. Hum Mutat. 2009; 30(9):1256-1266

15. Maltsev VA, ReznikovV, Undrovinas NA, Sabbah HN, Undrovinas A. Modulation of late sodium current by $\mathrm{Ca} 2+$, calmodulin, and CaMKII in normal and failing dog cardiomyocytes: similarities and differences. AmJ Physiol Heart Circ Physiol. 2008;294(4):H1597-H1608.

16. Wagner S, et al. Ca2+/calmodulin-dependent protein kinase II regulates cardiac $\mathrm{Na}+$ channels. JClin Invest. 2006;116(12):3127-3138.

17. Maier LS, Bers DM. Calcium, calmodulin, and calcium-calmodulin kinase II: heartbeat to heartbeat and beyond. J Mol Cell Cardiol. 2002;34(8):919-939.

18. Maier LS. Role of CaMKII for signaling and regulation in the heart. Front Biosci. 2009;14:486-496.

19. Hund TJ, et al. A $\beta_{\mathrm{IV}}$-spectrin/CaMKII signaling complex is essential for membrane excitability in mice. J Clin Invest. 2010;120(10):3508-3519.

\title{
In obesity and weight loss, all roads lead to the mighty macrophage
}

\author{
Alex Red Eagle ${ }^{1}$ and Ajay Chawla ${ }^{2,3}$
}

1Department of Genetics and 2Division of Endocrinology, Metabolism, and Gerontology, Department of Medicine, ${ }^{3}$ Graduate Program in Immunology, Stanford University School of Medicine, Stanford, California, USA.

\begin{abstract}
Obesity is associated with infiltration of white adipose tissue (WAT) by macrophages, which contributes to the development of insulin resistance. In this issue of the JCI, Kosteli and colleagues demonstrate that weight loss is unexpectedly also associated with rapid, albeit transient, recruitment of macrophages to WAT and that this appears to be related to lipolysis.
\end{abstract}

Macrophages are derived from monocytes and comprise a heterogenous population of cells found in nearly all tissues (1). In addition to their pivotal role in host defense, inflammation, and tissue repair, studies over the last decade have focused on their role in chronic metabolic diseases, such as obesity and insulin resistance (2). The initial descriptions of macrophage infiltration of white adipose tissue (WAT) during obesity, which were published in the JCI in 2003 $(3,4)$, garnered a great deal of attention, as evidenced by over 1,000 subsequent reports that confirmed the link between adipose tissue macrophages (ATMs) and inflammation and insulin resistance. In contrast, the role of innate and adaptive immunity in weight loss, the ultimate translational

Conflict of interest: The authors have declared that no conflict of interest exists.

Citation for this article: J Clin Invest. doi:10.1172/ JCI44721. goal of research on obesity, is less clear. In this issue of the JCI, Kosteli and colleagues report that acute weight loss results in recruitment of macrophages to WAT (5). However, in this case, the recruited macrophages do not promote inflammation but rather regulate lipolysis. Since stimuli that enhance adipocyte lipolysis increase macrophage recruitment to WAT, the authors suggest that release of FFAs is a general signal for macrophage recruitment.

\section{Obesity, inflammation, and macrophages}

The chronic, low-grade inflammation that is characteristic of obesity has long been suspected to contribute to the development of insulin resistance (6). Almost two decades ago, Spiegelman and colleagues demonstrated that TNF- $\alpha$, which is induced in the adipose tissue of obese animals, inhibits glucose disposal by promoting insulin resistance in peripheral tissues
(7). Although adipocytes were identified as the source of TNF- $\alpha$, expression of TNF- $\alpha$ was also observed in the stromovascular fraction rich in immune cells. This observation fueled a new direction in metabolic research and led to the discovery that macrophage infiltration of WAT is responsible for obesity-associated inflammation $(3,4)$.

While WAT from lean animals contains a resident population of alternatively activated macrophages (also known as M2 macrophages), which are characterized by expression of F4/80, CD301, and arginase 1 (Arg1), obesity is associated with recruitment of classically activated macrophages (also known as M1 macrophages), which are characterized by expression of F4/80, CD11c, and iNOS (Table 1). This observation led Lumeng and colleagues to conclude that obesity induces a switch in macrophage activation in WAT (8). Since alternatively activated macrophages can suppress inflammation in an autocrine and/or paracrine manner, in part via secretion of IL-10, the net effect of this shift in macrophage polarization is an increase in adipose tissue inflammation. However, this is likely to be an oversimplification because macrophages in vivo exhibit a 
Table 1

Phenotypic and functional heterogeneity of ATMs

\begin{tabular}{|c|c|c|c|c|}
\hline & Lean $^{A, B}$ & Obese $^{A, B}$ & Early weight loss/fasting ${ }^{A}$ & Late weight loss ${ }^{B}$ \\
\hline Origin & Resident & Recruited & Recruited & $\begin{array}{c}\text { Recruitment/ } \\
\text { turnover/egress (?) }\end{array}$ \\
\hline $\begin{array}{l}\text { Surface markers } \\
\text { (gene name) }\end{array}$ & 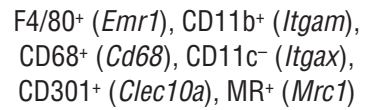 & 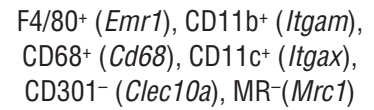 & $\begin{array}{l}\mathrm{F} 4 / 80^{+}(\text {Emr1 }) \\
\mathrm{CD}_{11 \mathrm{C}^{-}(\text {Itgax })}\end{array}$ & $\mathrm{CD}_{68}+(\mathrm{Cd} 68)$ \\
\hline Triggers & None & $\begin{array}{l}\text { Adipocyte death, ER stress, } \\
\text { tissue hypoxia, inflammation }\end{array}$ & Demand lipolysis & $?$ \\
\hline Phenotype & Alternative (antiinflammatory) & Classical (inflammatory) & $?$ & $?$ \\
\hline $\begin{array}{l}\text { Chemokine receptor/ } \\
\text { chemokine }\end{array}$ & $\begin{array}{l}\mathrm{CX}_{3} \mathrm{CR} 1(?) \\
\mathrm{CX}_{3} \mathrm{CL} 1(?)\end{array}$ & $\begin{array}{l}\text { CCR2 } \\
\text { CCL2 }\end{array}$ & $\begin{array}{c}\text { CCR2 } \\
?\end{array}$ & $\begin{array}{l}? \\
?\end{array}$ \\
\hline $\begin{array}{l}\text { Cellular product } \\
\text { (gene name) }\end{array}$ & $\begin{array}{c}\text { Arginase (Arg1), Ym1 (Chi3/3), } \\
\text { IL-10 (I/10) }\end{array}$ & $\begin{array}{c}\text { TNF- } \alpha \text { (Tnfa), IL-1 } \beta(/ / 1 b), \\
\text { NO (Nos2) }\end{array}$ & Antilipolytic factors (?) & IL-10 (I/10) \\
\hline $\begin{array}{l}\text { Metabolic } \\
\quad \text { consequences }\end{array}$ & $\begin{array}{l}\text { Insulin sensitivity/ } \\
\text { tissue homeostasis }\end{array}$ & Insulin resistance & $\begin{array}{l}\text { Suppress lipolysis/ } \\
\text { fasting response (?) }\end{array}$ & Insulin sensitivity \\
\hline
\end{tabular}

AMouse. BHuman.

dynamic range of gene expression patterns along the entire continuum of classical to alternative activation. Nonetheless, impairment in alternative macrophage activation, as in mice lacking either PPAR $\gamma$ or PPAR $\delta$ specifically in macrophages (9-11), leads to insulin resistance, whereas disruption of inflammatory pathways in classically activated macrophages confers protection against insulin resistance (12). Taken together, these findings suggest that a balance between alternatively and classically activated macrophages is required for normal adipocyte function and insulin action. Furthermore, in addition to macrophages, there is a growing list of immune cells, including $\mathrm{CD}^{+}$and $\mathrm{CD}^{+} \mathrm{T}$ cells, Tregs, and mast cells, that participate in the orchestration of inflammation in obese WAT (13-15).

\section{Macrophage recruitment during weight loss}

Based on the preferential recruitment of classically activated macrophages during obesity (Figure 1), weight loss would be predicted to have the opposite effect; i.e., reduction in adiposity should lead to reduction in ATM content and inflammation. This is indeed the case in patients that have undergone gastric bypass surgery for weight loss (16). However, these measurements were made three months after weight loss and only provide a snapshot of macrophage phenotypes in adipose tissue. To better define the role of macrophages in weight loss, Kosteli and colleagues established a model of gradual weight loss by reducing caloric intake to $70 \%$ of normal in mice made obese by high-fat diet (5). While chronic weight loss (>21 days after initiation of caloric restriction) led to a progressive decrease in ATM content in the WAT, there was a surprising increase in the number of ATMs during the first week of weight loss (Figure 1). Interestingly, despite the transient increase in ATMs during weight loss, the aggregate expression of markers of inflammation (Tnf, Nos2, Itgax [which encodes CD11c], Serpine 1) or alternative activation (Arg1, chitinase 3-like 3 [Chi3l3]) was not markedly different. However, definitive confirmation of this would require flow cytometric analysis of WAT macrophage subsets during weight loss.

Among the variables examined, increase in the breakdown of stored triglycerides in WAT was tightly correlated with recruitment of ATMs during weight loss (Figure 1). This observation led Kosteli and colleagues to postulate that fasting, which increases FFA release from adipocytes, might promote recruitment of macrophages to WAT (5). Indeed, administration of the highly selective $\beta 3$-agonist CL316,243, which induces lipolysis in adipocytes, or subjection of mice to a 24-hour fast led to an increase in ATMs. Conversely, inhibition of lipolysis by feeding of high-carbohydrate diet or genetic deletion of adipose triglyceride lipase (Pnpla2) prevented macrophage infiltration of WAT. Interestingly, macrophage chemotaxis was enhanced by adipose tissue explants from fasted mice, suggesting the release of a chemotactic factor. However, CCR2 ligands, which promote macrophage recruitment during obesity, were not differentially expressed, implicating the release of an unknown chemoattractant during fasting.

\section{ATM functions during weight loss}

While ATMs in obesity contribute to adipose tissue inflammation and insulin resistance, the functions of ATMs during weight loss are not known. To address this question, Kosteli et al. examined the morphological and molecular characteristics of ATMs during weight loss (5). These studies revealed remarkable similarities between weight loss ATMs and cholesterol-laden macrophages in atheromatous plaques. For instance, both sets of recruited macrophages exhibit higher expression of scavenger receptors (Cd36 and macrophage scavenger receptor 1 $[M s r 1])$ and lipid-handling genes (adipose differentiation-related protein $[A d f p]$, fatty acid-binding protein 4 [Fabp4], Apoe, and Abca1) and increased accumulation of oil red $\mathrm{O}$-positive neutral lipids. Despite these similarities, it is likely that the composition of lipid droplets found in ATMs during weight loss is distinct from that of atheroma macrophages; the latter are known to contain primarily cholesterol (17), whereas the former are likely engorged with triglycerides.

Based on the recruitment and gene expression characteristics of weight loss ATMs, one would predict that these cells facilitate the trafficking of lipids from adipose tissue to liver during fasting or acute weight loss. However, depletion of ATMs by clodronate-containing liposomes gave 


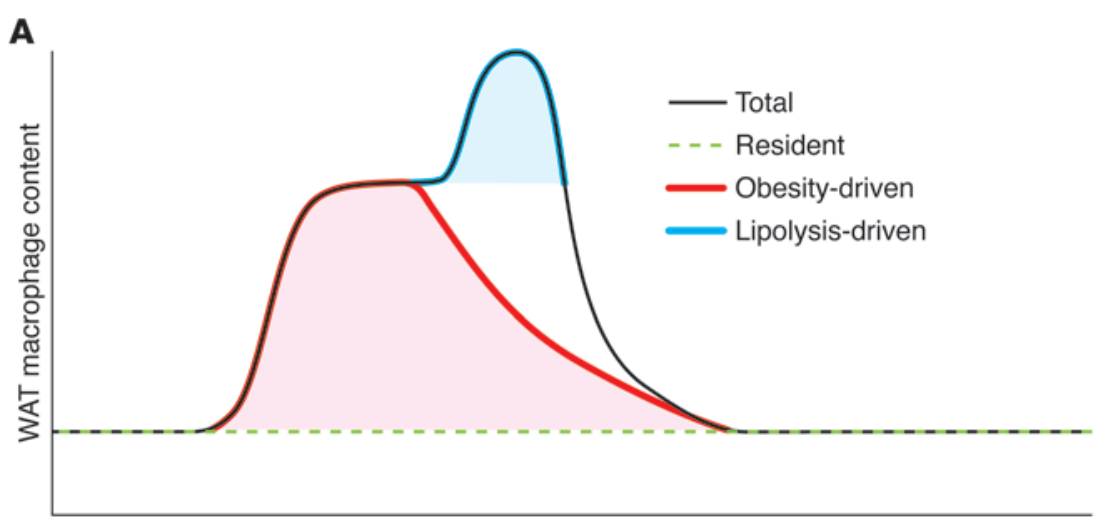

B

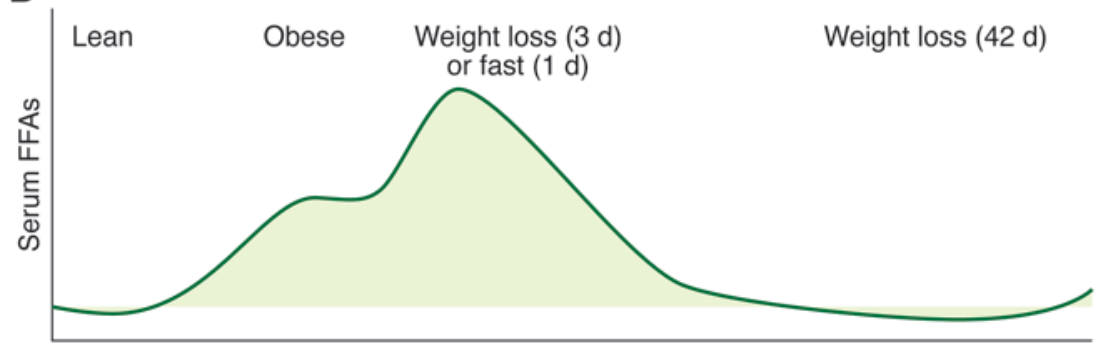

\section{Figure 1}

WAT macrophage content and adipose tissue lipolysis. (A) Adipose tissue contains a resident pool of macrophages (F4/80+CD11 ${ }^{-}$ CD301+). Obesity leads to the recruitment of inflammatory $\left(\mathrm{F} 4 / 80^{+} \mathrm{CD} 11 \mathrm{C}^{+} \mathrm{CD} 301^{-}\right)$macrophages, which are associated with insulin resistance. Early weight loss and fasting lead to the recruitment of an unexplored population of "antiinflammatory" macrophages $\left(\mathrm{F} 4 / 80^{+} \mathrm{CD} 11 \mathrm{C}^{-}\right)$. (B) The recruitment of this novel population correlates with release of FFAs and regulates adipose tissue lipolysis. the opposite result (5). The acute depletion of macrophages increased expression of Pnpla2, resulting in increased release of FFAs in fasted mice. Together, these findings suggest that the recruited ATMs function in a negative feedback loop to dampen the release of FFAs from WAT. How ATMs regulate adipose tissue lipolysis remains unknown and will be an important subject for future investigations.

\section{Concluding remarks}

The report by Kosteli et al. (5) is the first to describe the rapid recruitment of macrophages to adipose tissue during weight loss and their involvement in lipolysis (Figure 1). While broadening our understanding of the importance of the immune system in regulating metabolism, these initial studies also raise many new questions, specifically, the why, what, and how: why are macrophages recruited to WAT during fasting, what are the molecular signals that regulate their trafficking in WAT during weight loss, and how do the recruited ATMs regulate adipocyte triglyceride metabolism? We suggest that potential answers to these questions may reside in the known functions of macrophages in metabolism and host defense.

Our understanding of chronic metabolic diseases has been greatly informed by previous studies on macrophage involvement in the reverse cholesterol transport pathway, which promotes net movement of cholesterol from the periphery to the liver for its eventual degradation into bile acids (17). One can envision a similar role for ATMs during fasting or weight loss, in which they facilitate the net movement of FFAs from adipose tissue to liver for their eventual use in energy production. Thus, it will be important to understand the role of ATMs in metabolic adaptations to fasting and weight loss. In addition, the intracellular fate of FFAs taken up by fasting ATMs and the transcriptional response of macrophages to their influx needs to be thoroughly examined. Since fatty acids are endogenous activators of PPARs (2), the involvement of these nuclear receptors in weight loss ATMs deserves further exploration. Finally, the nature of chemoattractant signals (chemokines versus lipids) that promote recruitment of ATMs during fasting or weight loss needs to be investigated.

Because macrophages function as sentinels of host defense, functional consideration of weight loss ATMs in immunity is warranted. In this regard, recent studies have demonstrated dramatic tropism of dormant bacilli and parasites, such as Mycobacterium tuberculosis, Rickettsia prowazekii, and Trypanosoma cruzi, for adipose tissue, which allows these pathogens to evade host immunity (18-20). Since these pathogens cluster around adipocyte lipid droplets, lipolysis might release pathogen- associated lipids, providing an opportune moment for detection of these evasive pathogens. While many questions remain unanswered, the report by Kosteli and colleagues (5) suggests a crucial function for ATMs in both obesity and weight loss. Thus, similar to the road to obesity and insulin resistance, the road to better health might also involve macrophages.

\section{Acknowledgments}

We thank A. Loh for critique on this commentary. A. Chawla was supported by the NIH (DK076760, HL076746, DK081405), a Larry L. Hillblom Foundation Network grant, and the NIH Director's Pioneer Award (1DP1OD6415-1). A. Red Eagle was supported by the Stanford Medical Scientist Training Program and a Howard Hughes Medical Institute Gilliam fellowship.

Address correspondence to: Ajay Chawla, 300 Pasteur Dr., MC 5103, Stanford, California 94305-5103, USA. Phone: 650.724.4022; Fax: 650.725.7085; E-mail: achawla@stanford.edu.

1. Gordon S, Taylor PR. Monocyte and macrophage heterogeneity. Nat Rev Immunol. 2005;5(12):953-964.

2. Chawla A. Control of macrophage activation and function by PPARs. Circ Res. 2010;106(10):1559-1569.

3. Weisberg SP, McCann D, Desai M, Rosenbaum M, Leibel RL, Ferrante AW Jr. Obesity is associated with macrophage accumulation in adipose tissue. J Clin Invest. 2003;112(12):1796-1808.

4. $\mathrm{Xu} \mathrm{H}$, et al. Chronic inflammation in fat plays 
a crucial role in the development of obesityrelated insulin resistance. J Clin Invest. 2003 ; 112(12):1821-1830.

5. Kosteli A, et al. Weight loss and lipolysis promote a dynamic immune response in murine adipose tissue. J Clin Invest. 2010;120(10):3466-3479.

6. Shoelson SE, Lee J, Goldfine AB. Inflammation and insulin resistance. J Clin Invest. 2006; 116(7):1793-1801

7. Hotamisligil GS, Shargill NS, Spiegelman BM. Adipose expression of tumor necrosis factor-alpha: direct role in obesity-linked insulin resistance. Science. 1993;259(5091):87-91.

8. Lumeng CN, Bodzin JL, Saltiel AR. Obesity induces a phenotypic switch in adipose tissue macrophage polarization. J Clin Invest. 2007;117(1):175-184

9. Kang K, et al. Adipocyte-derived Th2 cytokines and myeloid PPARdelta regulate macrophage polarization and insulin sensitivity. Cell Metab. 2008;7(6):485-495.
10. Odegaard JI, et al. Macrophage-specific PPARgamma controls alternative activation and improves insulin resistance. Nature. 2007;447(7148):1116-1120.

11. Odegaard JI, et al. Alternative M2 activation of Kupffer cells by PPARdelta ameliorates obesity-induced insulin resistance. Cell Metab. 2008; 7(6):496-507.

12. Olefsky J, Glass C. Macrophages, inflammation, and insulin resistance. Annu Rev Physiol. 2010; 72:219-246.

13. Nishimura S, et al. CD8+ effector T cells contribute to macrophage recruitment and adipose tissue inflammation in obesity. Nat Med. 2009; 15(8):914-920

14. Winer $\mathrm{S}$, et al. Normalization of obesity-associated insulin resistance through immunotherapy. Nat Med. 2009;15(8):921-929.

15. Liu J, et al. Genetic deficiency and pharmacological stabilization of mast cells reduce diet-induced obesity and diabetes in mice. Nat Med. 2009;
15(8):940-945

16. Cancello R, et al. Reduction of macrophage infiltration and chemoattractant gene expression changes in white adipose tissue of morbidly obese subjects after surgery-induced weight loss. Diabetes. 2005; 54(8):2277-2286.

17. Yvan-Charvet L, Wang N, Tall AR. Role of HDL, $\mathrm{ABCA} 1$, and $\mathrm{ABCG} 1$ transporters in cholesterol efflux and immune responses. Arterioscler Thromb Vasc Biol. 2010;30(2):139-143.

18. Bechah Y, Paddock CD, Capo C, Mege JL, Raoult D. Adipose tissue serves as a reservoir for recrudescent Rickettsia prowazekii infection in a mouse model. PLoS One. 2010;5(1):e8547.

19. Combs TP, et al. The adipocyte as an important target cell for Trypanosoma cruzi infection. J Biol Chem. 2005;280(25):24085-24094.

20. Neyrolles O, et al. Is adipose tissue a place for Mycobacterium tuberculosis persistence? PLoS One. 2006;1:e43.

\title{
c-Maf and you won't see fat
}

\author{
Laurie K. McCauley
}

Department of Periodontics and Oral Medicine, School of Dentistry, and Department of Pathology, Medical School, University of Michigan, Ann Arbor, Michigan, USA.

\begin{abstract}
Osteoporosis is a common, age-related bone disease that results from an imbalance between the processes of bone formation and bone resorption, resulting in reduced bone mass and increased risk of fracture. Mesenchymal stem cells have the capacity to differentiate into osteoblastic and adipogenic lineages; recent research suggests that the switch between these two fates may be key to the decreased bone density that occurs with aging. In this issue, Nishikawa et al. demonstrate that the basic leucine-zipper transcription factor Maf (also known as c-Maf) is central to osteoblast lineage commitment. In addition, they find that increased oxidative stress - as occurs with aging - decreases Maf expression. This work advances understanding of the transcriptional regulation of cell fate decisions and may help direct the development of new therapies to fight age-related bone loss.
\end{abstract}

Osteoporosis is a metabolic bone disease that results from an imbalance between the processes of bone formation and bone resorption, leading to reduced bone mass and increased susceptibility to fracture. Osteoporosis is commonly age associated, and one out of two women and one out of four men over the age of 50 will experience an osteoporotic fracture during their lifetime, with patient care costs estimated at $\$ 14$ billion per year $(1,2)$. Recent research has identified a correlative relationship of fat deposition in bone marrow with bone density. Many but not all studies link the two in an inverse relationship that suggests marrow fat may be driven at the

Conflict of interest: The author has declared that no conflict of interest exists.

Citation for this article: J Clin Invest. doi:10.1172/ JCI44786. expense of new bone formation. While the role of fat in bone remains unclear, current thinking supports a reciprocal relationship between adipogenesis and osteogenesis governed by mesenchymal stem cell (MSC) lineage allocation.

The bone marrow houses a wide variety of cell types at various stages of differentiation. Cells of the mesenchymal lineage reside in the marrow and give rise to osteoblasts and adipocytes, among other cell types (3). Over the past decade, a considerable effort has been devoted toward understanding how MSC fates can be directed toward adipogenic and osteogenic lineages. The critical determinants of this switch are unclear, but a complete understanding of the process may direct the development of therapies for age-associated bone loss. The mutual exclusivity of the osteoblast and adipocyte cell fates suggests that sig- nals that direct cells down one lineage may prevent them from traversing down the other. Prime examples of these switch signals are CCAAT/enhancer binding protein (C/EBP), a trigger for adipogenesis; PPAR $\gamma$, which promotes adipocyte maturation; and Runx2, an osteoblastic transcriptional mediator (4). Impaired PPAR $\gamma$ signaling shifts the fate of MSCs in the marrow toward the osteoblast lineage (5). The wnt pathway can suppress PPAR $\gamma$, favoring MSC differentiation to osteoblasts (6). Other transcriptional mediators associated with osteoblast/adipocyte specification include $\Delta \mathrm{FosB}, \mathrm{TAZ}$, Esr1, Msx2, C/EBP $\beta$, and Id4, though their specificity as determinants of the age-related switch in bone is unclear $(7,8)$.

\section{Identification of Maf as a cell fate switch factor}

In this issue, Nishikawa et al. demonstrate, in an elegant series of studies, that the basic leucine-zipper (bZIP) transcription factor Maf (musculoaponeurotic fibrosarcoma, also known as c-Maf) is central to osteoblast lineage commitment with age (9) (Figure 1). Via a genome-wide screening of transcription factors expressed in cells derived from calvaria, Maf was identified as a factor that increased more than 4-fold during osteoblastogenesis. It was further found to be highly expressed in bone marrow stromal cells (BMSCs) and reduced in 